\title{
A double blind randomized clinical trial to compare the efficacy of bupivacaine and ropivacaine for painless delivery
}

\author{
Maryam Shokrpour $1 \square$, Narges Anousheh $2 \square$, Shirin Pazouki ${ }^{3} \square$, Alireza Kamali ${ }^{3^{*}}$ \\ Author affiliations: \\ 1. Department of Gynecology, Arak University of Medical Sciences, Arak, Iran. \\ 2. Medical Sciences Research Committee, Arak University of Medical Sciences, Arak, Iran. \\ 3. Department of Anesthesiology and Critical Care, Arak University of Medical Sciences, Arak, Iran. \\ *Correspondence: Alireza Kamali, Department of Anesthesiology and Critical Care, Arak University of Medical Sciences, Arak, \\ Iran; Email: alikamaliir@yahoo.com; Tel: 00989181622810
}

\section{Abstract}

Background \& objective: Childbirth is an important phenomenon in the life of the mother and although this experience is the wish of every mother, it also concerns her due to the labor pain and the possible dangers that threaten her. There are several medications for painless delivery, such as epidural analgesia with the help of bupivacaine and ropivacaine. The aim of this study was to compare the efficacy of painless delivery with epidural method by bupivacaine and ropivacaine in women candidates for painless delivery.

Methodology: This study is a double blind randomized clinical trial. In this study, patients were randomly divided into two equal groups of bupivacaine and ropivacaine using cubic random method. The demographic data, incidence of cesarean section and mean birth phases were evaluated. Finally, data were analyzed using SPSS 22 software, ANOVA and T-test.

Results: A total of 112 patients were enrolled in the study. There was no significant difference between the two groups in terms of mean active phase of labor, mean Apgar score of min 1 and 5, incidence of cesarean section and mothers motor block score, but the mean of the second phase of labor and mean use of delivery helping instruments (such as forceps and vacuum) were lower in the ropivacaine group. Also, the mean score of patients' satisfaction was higher in ropivacaine group.

Conclusion: It could be concluded that the combination of ropivacaine and fentanyl versus the combination of bupivacaine and fentanyl was a very suitable combination for controlling labor pain by epidural method, while it did not increase the rate of cesarean section and did not have a negative effect on neonatal Apgar scores of min 1 and 5 , it had a significant effect on decreasing the mean of second stage of labor, decreasing the use of delivery helping instruments and increasing patient satisfaction score.

Key words: Bupivacaine; Ropivacaine; Epidural analgesia; Painless delivery

Citation: Anousheh N, Shokrpour M, Pazouki S, Kamali A. A double blind randomized clinical trial to compare the efficacy of bupivacaine and ropivacaine for painless delivery. Anaesth. pain intensive care 2021;25(3): 274-279.

DOI: $10.35975 / a p i c . v 25 i 3.1146$

Received: November 2, 2020, Reviewed: February 22, 2021, Accepted: March 26, 2021

\section{Introduction}

Childbirth is an important phenomenon in the life of a mother ${ }^{1}$ and although this experience is the wish of every mother, it is also followed by her concern over the pain of childbirth and the potential dangers that threaten her. ${ }^{2}$ Labor pain is one of the most 
excruciating pain that women experiences in their lifetime. ${ }^{3}$ Several studies have considered it to be the most severe pain, such as amputation. ${ }^{4}$ Severe labor pain has adverse effects on the mother and fetus, including increased cardiac output, increased blood pressure and mother's heart rate, decreased contractility, slow delivery, decreased placental blood flow, and limited oxygen supply to the fetus. ${ }^{5}$ The most common reason for choosing cesarean section was fear (48.3\%) and maintaining fetal health $(18.3 \%)$. The most common cause of fear is fear of labor pain and the absence of measures to relieve it. ${ }^{6}$ Therefore, the emphasis has always been on using different methods of painful delivery to increase mothers' satisfaction and reduce the complications of childbirth. Today, labor analgesia is performed in a variety of ways, including psycho-prophylaxis TENS, systemic drug administration, inhalation techniques, neural axis blocking, and techniques such as caudal block or para-servical block at a more limited level. Spinal anesthesia and epidural anesthesia or a combination of the two may cause more complete analgesia. $^{7}$

Epidural anesthesia is one of the most popular methods of inducing labor pain with central nerve block used by local anesthesia close to the nerves that are widely used to relieve pain. ${ }^{8}$ Drugs used for epidural anesthesia include fentanyl, bupivacaine and ropivacaine.

Fentanyl is a potent opiate drug that is 75 times more potent than morphine and is used to induce analgesia and anesthesia in various ways. ${ }^{9}$

Bupivacaine is one of the topical anesthetic drugs of the amide group with a mechanism to inhibit the passage of sodium through specific sodium channels and nerve impulse blocks. As the sodium permeability decreases, it results in a depolarization of the nerve and is easily stimulated by the decrease. It is one of the long-acting drugs with concentrations of $0.5 \%$ and $0.25 \%$ and its maximum dose without epinephrine is $175 \mathrm{mg}$ gram and $225 \mathrm{mg}$ with epinephrine. ${ }^{10}$

Ropivacaine can be considered an amide topical anesthetic that is similar to bupivacaine and has a lower block of movement than bupivacaine, so it may allow the patient to be discharged earlier from Recovery. ${ }^{11}$
The aim of this study was to determine the efficacy of painless delivery by two methods of bupivacaine and ropivacaine in women candidates for painless delivery.

\section{Methodology}

This study is a randomized, double blind clinical trial for all pregnant women referred to Taleghani Hospital, Arak, Iran, who were candidates for natural delivery who met the inclusion criteria. In all stages of research, all ethics statements in Helsinki Research and Research Committees in Arak University of Medical Sciences were considered. Code of Ethics IR.ARAKMU.REC.1397.134 was adopted on $8 / 19 / 2019$. The registration code at the Iran Clinical Trial Center is IRCT20180903040936N1.

Inclusion criteria included ASA grades II, full term (37-42 weeks gestation) mothers, with singleton pregnancy, who gave informed consent to participate in the study.

All patients in epidural group in which epidural block failed, and the parturients who had to undergone caesarean section for emergency reasons (such as placental abruption, umbilical cord prolapse, etc.) were excluded.

Only the anesthesiologist in charge of the study was aware of the type of study and the study groups, while the injectable drug type were concealed from the patients. Also, the project intern in charge of filling out the questionnaires was not aware of the groups according to the injectable drug and only knew the groups based on A and B and filled out the questionnaires accordingly.

The patients were randomly divided into two equal groups of epidural anesthesia either with bupivacaine or ropivacaine, using cubic random method. Thus, 56 parturients undergoing painless delivery (epidural anesthesia) were treated with bupivacaine. Parturients were administered 3-5 ml/kg of crystalloid fluids. In sitting position they underwent epidural anesthesia with $0.125 \%$ bupivacaine (4-6 ml) and $5 \mu \mathrm{g}$ fentanyl through spinal needle 20G, at L3-L4 or L4-L5 space. An epidural catheter was fixed. For maintenance dose, a combination of $0.125 \%$ bupivacaine and $0.002 \%$ fentanyl at $6-10 \mathrm{ml} / \mathrm{h}$ was used. 


\begin{tabular}{l|ll|l}
\hline \multicolumn{3}{l}{ Table 1: Comparison of age and gestational age of mothers who candidates for painless delivery } \\
\hline Group/Age and gestational age & Bupivacaine group & Ropivacaine group & $p$-value \\
\hline Average age & $2.6 \pm 1.24$ & $2.1 \pm 2.25$ & $p=0.4$ \\
\hline Average gestational age & $3.7 \pm 2.38$ & $6.1 \pm 2.39$ & $p=0.6$ \\
\hline
\end{tabular}

\begin{tabular}{llll}
\hline \multicolumn{4}{l}{ Table 2: Comparison of the mean Apgar scores of min $\mathbf{1}$ and 5, in candidates for painless delivery } \\
\hline Group/Apgar score & Bupivacaine group & Ropivacaine group & $p$ - value \\
\hline Mean Apgar score at min 1 & $8.85 \pm 0.95$ & $8.91 \pm 0.77$ & $p=0.4$ \\
\hline Mean Apgar score at min 5 & $9.83 \pm 1.1$ & $9.79 \pm 0.98$ & $p=0.4$ \\
\hline
\end{tabular}

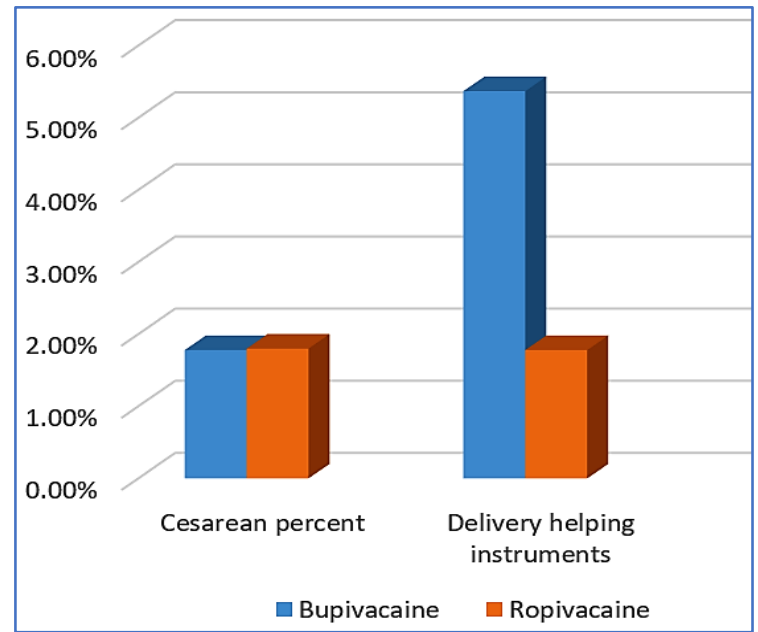

Figure 1: Comparison of the incidence of cesarean section and the rate of use of helping insrumes

In the second group, considered as ropivacaine group, after obtaining informed consent, 56 pregnant women who were candidates for natural delivery underwent painless delivery (epidural anesthesia) with ropivacaine. Patients in this group received a $16 \mathrm{mg}$ ampoule of ropivacaine $(8 \mathrm{ml})$ and $1 \mathrm{ml}$ opioid, equivalent to $500 \mu \mathrm{g}$ of fentanyl, which we brought to $12 \mathrm{ml}$ with distilled water.

Sample size:

$\mathbf{n}=\frac{(z 1-\alpha / 2+z 1-\beta)^{2}(\delta 1+\delta 2)^{2}}{(\mu 1-\mu 2)^{2}}$

$\mathrm{z} 1-\alpha / 2=1.96$

$\mathrm{z} 1-\beta=1.28$

$\delta 1=77 \mu 1: 267$ $\mu 2=212$

$\delta 2: 51$

$\mathrm{n} \cong 56$

$\mathrm{N}=56 \times 2=112$

Statistical analysis: For both groups, a questionnaire containing demographic information, incidence of cesarean section and mean delivery phases was completed. Finally, data were analyzed using SPSS 22 software and ANOVA and T-test was statistically analyzed.

\section{Results}

A total of 112 patients were included in the study based on inclusion and exclusion criteria and were divided into two groups: ropivacaine and bupivacaine. In Table 1, the comparison of age and gestational age of mothers who were candidates for painless delivery in the two groups of bupivacaine and ropivacaine were evaluated and the mean age $(\mathrm{P}$-value $=0.4)$ and mean gestational age $(\mathrm{P}$-value $=0.6)$ were compared between the two groups. No significant difference was observed and the mean age of both groups was 25 years and mean gestational age was 38.5 weeks.

The mean duration of active phase (in hours) in the bupivacaine and ropivacaine groups were $3.1 \pm 0.78$ and $3.04 \pm 0.28$ respectively and phase II (in $\mathrm{min}$ ) in the mothers candidate for painless delivery in the two groups were $52.8 \pm 4.9$ and $39.7 \pm 4.6$ respectively,

There was no significant difference between the two groups in terms of mean active phase of labor $(p=0.4)$ and the average active phase of labor was approximately 3 hours in both groups. However, there was a significant difference between the two groups in terms of mean phase II. But there was a significant difference between the two groups regarding the mean 
of the phase II of labor ( $p=0.01)$. Significantly, the mean of the second phase of labor was lower in the ropivacaine group.

Comparing the mean Apgar scores of min 1 and 5 of neonates, the mothers candidates for painless delivery in the two groups of bupivacaine and ropivacaine were compared in Table 2 and the results showed that between the two groups, the mean of Apgar scores of min 1 and 5, there was no significant difference was observed in painless delivery $(\mathrm{p}=0.4)$. In both groups, the mean Apgar score of min 1 was 8.8 and min 5 was 7.9 .

The percentage of cesarean section in the two groups was $1.78 \%$ and $1.80 \%$, respectively. The mean use of delivery helping instruments (such as forceps and vacuum) was $5.37 \%$ and $1.78 \%$, respectively. There was no significant difference between the two groups regarding the incidence of cesarean section in the mothers candidates for painless delivery $(p=0.4)$, the incidence of cesarean section in both groups was $7.1 \%$.

But there was a significant difference between the two groups regarding the use of delivery helping instruments (such as forceps and vacuum) and the mean use of these devices was significantly lower in the ropivacaine group $(\mathrm{p}=0.01)$.

The mean scores of satisfaction for mothers in the bupivacaine group and ropivacaine group were. \pm 0 52.67 vs. $2.3 \pm 0.86$ ( $p=0.02$ ) respectively. The results showed that there was a significant difference between the two groups in the mean score of satisfaction of mothers who were candidates for painless delivery, the mean score of satisfaction was higher in the ropivacaine group $(\mathrm{P}$-value $=0.02)$.

Regarding mean motor block score, in mothers who were candidates for painless delivery in the bupivacaine group was $2.76 \pm 0.78$ and in the ropivacaine group was $2.92 \pm 0.81$; according to these results no significant difference was observed between the two groups.

\section{Discussion}

In the world, about 200 million pregnancies occur each year, mostly resulting in live births. But for some women, delivery is not satisfactory and is associated with pain, fear, and suffering. ${ }^{12}$ Labor pain is one of the most severe pain women experience in their lives, with 35 to $58 \%$ of women describing it as unbearable. ${ }^{13}$ Obtaining the right combination for epidural anesthesia is one of the important goals of anesthesiologists and gynecologists while giving birth is a physiological process and always emphasizes normal delivery over cesarean section, but with severe pain that can be unbearable to the mother and even harmful to the fetus. ${ }^{14,15}$ Epidural anesthesia has been widely accepted as the most effective pain relief method in recent decades, while the choice of drug combination used in epidural anesthesia is important, so this study compared two drug compositions, bupivacaine and ropivacaine.

The results of this study showed that the mean of phase II of labor was significantly lower in ropivacaine group. In a study conducted by Kamali et al, in 2017, the delivery phases of painless delivery were compared with epidural, general analgesia with Entonox and the control group. The results of this study indicated that painless delivery techniques such as entonox and epidural did not increase the duration of the active and second phase of labor but rather use of entonox could significantly decrease the duration of the second phase of labor. ${ }^{1}$ The results of our study were consistent with this study, because in our study the two drug combinations used in epidural anesthesia did not have a different effect on the active phase of labor and the mean active phase of labor was similar in both groups. However, the mean of the second stage of labor was lower in the ropivacaine group.

In a 2015 study by Shanbin Guo et al., They compared epidural anesthesia to reduce labor pain with two combinations of bupivacaine and ropivacaine, the results of a meta-analysis of 50 clinical trials and motor block was more frequently reported in the bupivacaine group. Delivery helping instruments, oxytocin induction and cesarean delivery were also reported. ${ }^{16}$ The results of this study were almost in line with our study, because in our study the rate of cesarean section and mean active phase of labor in the two groups were similar. However, in our study the motor block was similar in the two groups and the use of delivery helping instruments was less frequently reported in the ropivacaine group. Also, the satisfaction score in the ropivacaine group was higher 
in our study than in the bupivacaine group. In this study, there was a significant difference between the two groups in the mean score of satisfaction for mothers who were candidates for painless delivery and the mean score of satisfaction was higher in the ropivacaine group.

In another study by T. N. Chethanananda et al., 2017, they compared the efficacy of the minimum concentration of ropivacaine and bupivacaine for painless delivery by the epidural method. The results of this study, which were performed on 60 mother candidates for analgesia, showed no significant difference in pain score, motor block and satisfaction score in the two groups. ${ }^{17}$ The results were inconsistent with our study because in our study the mean of second stage of labor in the ropivacaine group was lower than the bupivacaine group and the mothers' satisfaction in using the ropivacaine group was higher than the bupivacaine group. The cause of this difference may be due to the dose of the compounds used in painless delivery by epidural method.

Also, in this study, mean Apgar scores of min 1 and 5 were not significantly different between the bupivacaine and ropivacaine groups. In a 2011 study by Millicent Anim Somuah et al., They investigated painless delivery without epidural anesthesia, and concluded that epidural anesthesia was highly effective in reducing labor pain and had no negative effect on increased cesarean section or postpartum back pain and headache. Also in neonatal Apgar scores, there was no decrease in this method. ${ }^{18}$ The results in this study were somewhat consistent with our study, as in our study, both groups had analgesia and no adverse effects on Apgar score of 1 and 5 mins, respectively. Also, there was no significant difference between the two groups in the rate of cesarean section.

\section{Conclusion}

Comparison of our study with our previous studies indicates that the combination of ropivacaine and fentanyl versus the combination of bupivacaine and fentanyl was a very good combination for controlling labor pain in the epidural method, while not increasing the rate of cesarean section and having no negative effect on Apgar score. But it had a very positive effect on reducing the mean of the second stage of labor, reducing the use of delivery helping instruments and increasing patient satisfaction scores. Ropivacaine is a suitable alternative to bupivacaine in painless delivery with epidural anesthesia.

\section{Conflict of interest}

Nil declared by the authors.

\section{Authors' contribution}

All authors took part in conduct of the study, literature search and manuscript preparation.

\section{References}

1. Kamali A, Shokrpour M, Mashhadi E, Morahem MH. Comparison of labor phases in painless delivery with epidural analgesia and entonox administration. International Journal of life sciences \& Pharma research. 2017; 7(2). 155161. [Free full text]

2. Grigg $L$, Day $R$. Using remifentanil in labour via patientcontrolled analgesia. Prof Nurse. 2003 Sep;19(1):45-7.. [PubMed]

3. Rooks JP. Use of nitrous oxide in midwifery practice-complementary, synergistic, and needed in the United States. J Midwifery Womens Health. 2007;52(3):186-9. [PubMed] DOI: 10.1016/j.jmwh.2007.02.017

4. Esfandiari Marzieh, Nankaley A.A.D., Sanjari N., Almasi Afshin, Karimi Sedigheh. Effectiveness Of Entonox On Severity of labor pain in women referred to maternity ward of imam reza hospital, kermanshah, 2007. Journal Of llam University Of Medical Sciences. 2009 [cited 2021May26];17(1):25-30. Available from: https://www.sid.ir/en/journal/ViewPaper.aspx?id=]144506

5. Ji X, Qi H, Liu A. Clinical study on labor pain relief using the combined spinal-epidural analgesia and inhaling nitrous oxide. Zhonghua Fu Chan Ke Za Zhi. 2002;37(7):398-401. [PubMed]

6. Hasanzahraei $\mathrm{R}$, Mehran $\mathrm{N}$, Fathizadeh $\mathrm{N}$, Abedi $\mathrm{H}$. Epidural painless delivery: a phenomenology research. Iranian Journal of Nursing and Midwifery Research. 2007;12(1):25-9. [ResearchGate]

7. Goodman P, Mackey MC, Tavakoli AS. Factors related to childbirth satisfaction. J Adv Nurs. 2004;46(2):212-9. [PubMed] DOI: 10.1111/j.1365-2648.2003.02981.x

8. MHeinze S, Sleigh M. Epidural or no epidural anaesthesia: relationships between beliefs about childbirth and pain control choices. Journal of reproductive and infant psychology. 2003;21(4):323-33. [PubMed] DOI: 10.1080/02646830310001622132

9. Nejad A, Muslim N, Hossein S. Comparison of the effect of epinephrine, fentanyl and sufentanil on spinal anesthesia with lidocaine. The Horizon of Medical Sciences. 2007;13(3):5-12. [Free full text] 
10. Mehrdad H, Ismail Ak, Khosro Sh. Comparison the effect of subcutaneous injection of bupivacaine $0.5 \%$ before and after appendectomy on postoperative pain. Iranian Journal of Surgery. 2009;17(1):250-9. [PubMed]

11. Ateser RY, Kayacan N. Intrathecal ropivacaine in cesarean delivery. Niger J Clin Pract. 2017 Oct;20(10):1322-1327. [PubMed] DOI:10.4103/1119-3077.219520

12. Hasanzahraei $R$, Mehran N, Fathizadeh N, Abedi HA. Epidural painless delivery: A phenomenology. research. IJNMR 2007;12(1): 25-31. [PubMed]

13. Goodfellow CF, Hull MGR, Swaab DF, Dogterom J, Buijs RM. Oxytocin deficiency at delivery with epidural analgesia. $\mathrm{Br} J$ Obstet Gynecol. 2005;90(8):214-9. DOI: /10.1111/j.1471-0528.1983.tb08611.x

14. Kalra S, Saraswat N, Agnihotri G. Comparison of efficacy of bupivacaine and fentanyl with bupivacaine and sufentanil for epidural labor analgesia. Saudi J anaesth. 2010;4(3):178. [PubMed] DOI: 10.4103/1658-354X.71569

15. Lv BS, Wang W, Wang ZQ, Wang XW, Wang JH, Fang F, Mi WD. Efficacy and safety of local anesthetics bupivacaine, ropivacaine and levobupivacaine in combination with sufentanil in epidural anesthesia for labor and delivery: a meta-analysis. Curr Med Res Opin. 2014 Nov;30(11):227989 [PubMed] DOI: 10.1185/03007995.2014.946127

16. Guo S, Li B, Gao C, Tian Y. Epidural Analgesia With Bupivacaine and Fentanyl Versus Ropivacaine and Fentanyl for Pain Relief in Labor: A Meta-Analysis. Medicine (Baltimore). 2015 Jun;94(23):e880. [PubMed] DOI: 10.1097/MD.0000000000000880

17. Chethanananda TN, Shashank MR, Madhu N, Achyutha J, Siva Kumar KV. Comparative Efficacy of Minimal Concentration of Racemic Bupivacaine $(0.0625 \%)$ with Fentanyl and Ropivacaine (0.1\%) with Fentanyl for Epidural Labor Analgesia. Anesth Essays Res. 2017 JulSep;11(3):583-588. DOI: 10.4103/aer.AER 63_17

[PubMed]

18. Anim-Somuah M, Smyth RM, Cyna AM, Cuthbert A. Epidural versus non-epidural or no analgesia for pain management in labour. Cochrane Database Syst Rev. 2018 May 21;5(5):CD000331.

DOI: 10.1002/14651858.CD000331.pub4 


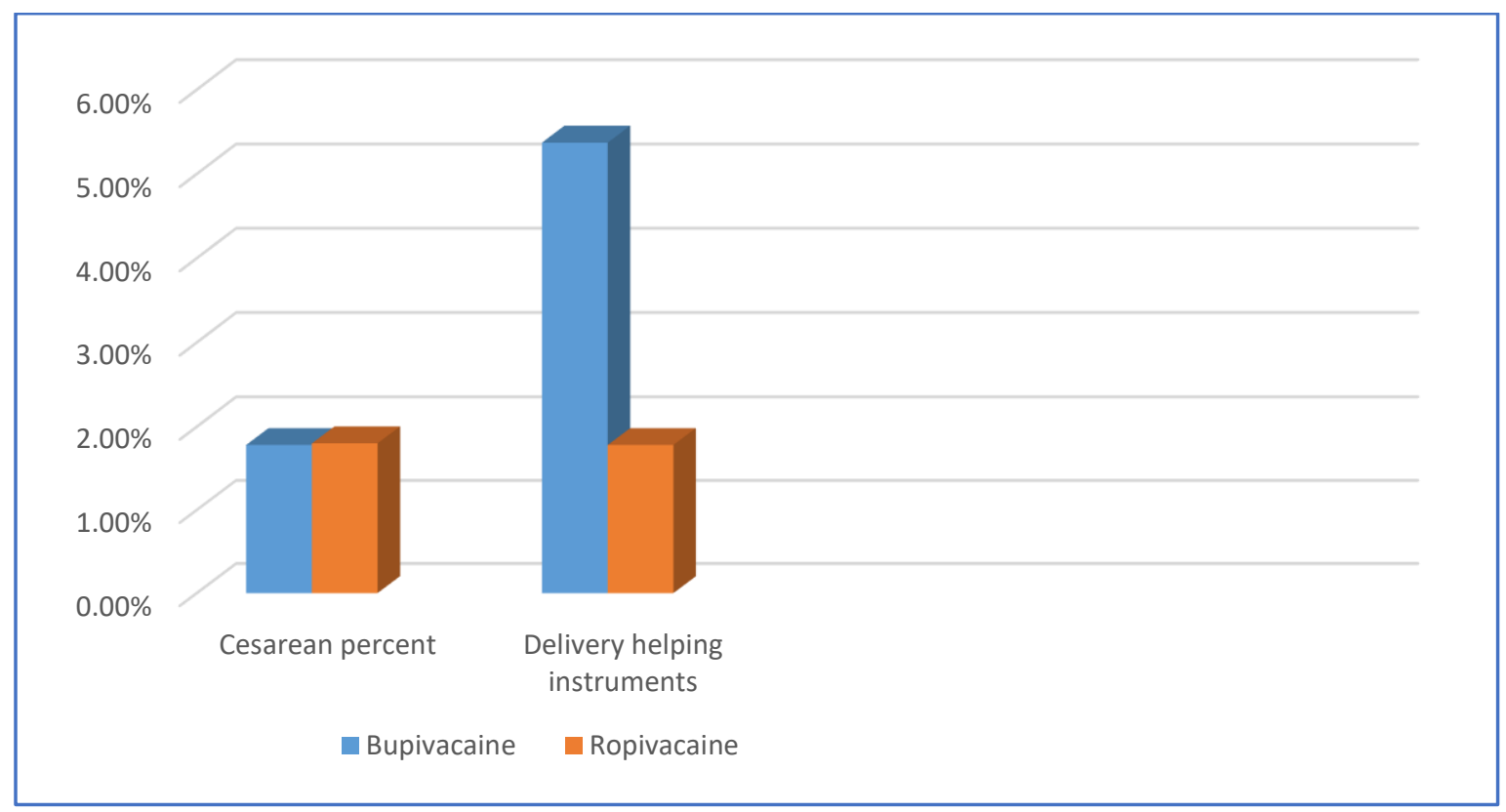

Figure 1: Comparison of the incidence of cesarean section and the rate of use of delivery helping instruments 